\title{
The 'social' machine: the computer as a participant in social and cognitive interactions within the classroom
}

\author{
Carolyn Dowling \\ Australian Catholic University \\ Victoria \\ Australia
}

\begin{abstract}
Computers play a multiplicity of roles in today's classrooms. Some of these are rightly perceived as a matter of choice, relating clearly to prevailing educational aims and philosophies, to the intentions of the teacher and to the manner in which these can be realized through the resources available. Others, however, appear to be an inevitable consequence of certain characteristics of our broader conceptions and experiences of computing technology. This paper examines the degree to which the 'psychological' dimension of the computer leads it to function as a 'social' entity within the classroom participating at a number of levels, beyond the deliberate intentions of the teacher, in the social and cognitive relationships and interactions which mediate learning. The contribution of the human/computer metaphors associated with traditional research in artificial intelligence is considered in combination with some of the implications of the use of language in human computer interfaces.
\end{abstract}

Main conference themes: implications

Educational areas:

Study topics:

Secondary keywords: cognition, culture, future developments, human computer interface 


\section{THE: SOCIAL MACHINE}

Computers play a multiplicity of roles within today's classrooms. Most of these we customarily perceive as being a matter of choice, relating clearly to prevailing educational aims and philosophies, to the intentions of the teacher, and to the manner in which these intentions can be realized through the hardware and software available. The decision as how the computer is to be used within a particular context, for instance as an information source, as a tutor, as a stimulus to discussion, and so on, is to a large extent under the control of individual teachers. There are also, however, computer roles which have not been specifically designed or intended by either the teacher or the software developer. These are the consequence of more general perceptions about computers which exist within contemporary society, beyond the bounds of specifically educational settings. One of these is the degree to which the 'psychological' dimension of the computer leads it to function as a 'social' entity within the classroom, participating at a number of levels beyond the deliberate intentions of the teacher in the interpersonal relationships and interactions which mediate learning.

\section{The 'personal' computer}

The concept of the 'personal' computer is one which undergoes continuous change as computing technology and our understanding of its inherent possibilities develop. Where once individual ownership of hardware was sufficient to characterize computers as 'personal', the intimacy with which many users relate to the technology has led to more complex conceptions concerning the interdependence of human beings and computers. These conceptions include the image of a cyborg, a composite entity consisting of various configurations and combinations of human 'meat', and the intellectual capacities of the computer. This is a conception which has long fascinated writers of science fiction and which has more recently become a topic for academic discussion in a number of areas including feminist theory [1]. While in some instances the fusion is perceived as being literal and physical, in others the conception is more subtle, alluding to existing partnerships of person and machine engaged in tasks which would be beyond the capability of either alone. A further sense in which computers might be characterized as personal, relates to the degree to which humans and computers can be said to engage in arguably social relationships incorporating significant elements of human to human interactions. This possibility may be fruitfully explored through an examination of aspects of the personification of the computer which apply both in classroom settings and in broader cultural contexts. 
Over many years the metaphorical understandings fostered in particular by the imagery of the early work in artificial intelligence in which aspects of human thought processes are understood in terms of computing, and vice versa, have contributed to wide acceptance of the notion that computers share with human beings what has traditionally been one of the defining characteristics of humanity, namely intelligence. As described by MacCormac [2]:

Under the computational metaphor, the brain can be viewed as a computational device similar to a computer, and the mind emerges as a series of programs by means of which the brain functions. Human thinking does not necessarily reduce to brain functions; rather, human thinking and brain functions combine to produce a computational process. The 'hardware' of the brain operates under the control of the 'software' of the mind to produce a computation which has traditionally been called cognition (p. 158).

While many writers caution against a too literal interpretation of this image, the extent to which it has grasped the public imagination is well reflected in popular culture, as well as in more academically oriented discourse, particularly in the area of cognitive science where this analogy has provided a model of human thinking which can be embodied in and investigated through the operations of computers. As Winner puts it [3]:

When we use terms like output, feedback, interface, and networking to express the transactions of everyday life, we reveal how thoroughly artificial things now shape our sense of a human being. As we compare our own minds to the operations of a computer, we acknowledge that an understanding of technical devices has somehow merged with the most intimate levels of selfunderstanding. Seldom, however, are such matters the subject of critical reflection (p. ix).

\section{The 'talking' computer}

A further aspect of computing technology which contributes substantially to an acceptance of computers as significantly personified entities, is the extent to which language is a feature of the vast majority of human computer interfaces. As with the concept of intelligence the use of language has traditionally been a key attribute which characterizes human beings and distinguishes them from other living things. Thus the language of the interface can be seen as a quality or capability which places user and computer in the same category. It is well accepted that even a shallow appearance of natural language use conveys sufficient sense of 
personification for the computer's role to become that of participant in a social interaction or relationship.

Although there is some dispute among software critics concerning the advisability of having 'personalities' in computer programs, their presence seems unavoidable. Any time there is communication between a computer and a human, the information presented by the computer has a certain style, diction, and tone of voice which impact upon the human's attitude and response toward the software $[4$, p. 320].

Winner argues in similar vein that the acceptance of personification is easy for us, particularly where language is involved, in that "We carry with us highly structured anticipations about entities that appear to participate, if only minimally, in forms of life and associated language games that are parts of human culture" [3, p. 14]. Dennett also discusses the way in which, what he terms the psychological 'thinness' of AI programs, is masked very effectively by their apparent use of language in a 'human' way [5, p. 115].

Although the degree to which computers are perceived as being in differing degrees personified varies considerably, it can be argued strongly that such qualities are never entirely absent. In addition, however, to this inevitable and, in terms of many purposes, inadvertent personification differing degrees of anthropomorphism are deliberately implemented in many computer applications including some designed for classroom use. An interesting example is that of Logo where this personification occurs at a range of levels for a variety of purposes. Solomon, for instance, writes [1]:

It helps to visualize these programs as scripts which can be read and carried out by any one of a large population of helpers residing in the machine. These people inside the computer do the work as instructed in the program. A program can sometimes be seen as a script, sometimes as a little person, and sometimes as an army of little people ... Anthropomorphizing is helpful in teaching children and is used frequently in different ways in this culture. Our metaphors are based on developing animate attributes of computers and computational ideas (p. 120).]

\section{Anthropomorphism and Logo}

Central to most implementations of Logo is the Turtle, an initial sense of its animation being suggested by its name and by the fact that it moves. As an object it possesses both quantitative and qualitative properties connected with its ability to move and with its state prior to and subsequent to movement. As has been pointed out by Piaget and many others, movement is a key factor in the distinction made by young children between the living and the nonliving. This is of importance within the Logo environment in establishing a foundation 
for the links between the Turtle's movements and those of the student which links enable so-called 'syntonic learning' to take place.

The Turtle is also a user of language. It responds to commands, albeit in a computer 'language', and is wont under special circumstances to address the user in grammatically impeccable natural language utterances. As Turkle [7] has pointed out computational objects differ from those considered by Piaget in their capacity to interact to varying degrees through language. This changes the focus of the categorization of animate/inanimate from movement to questions of thought and feeling - that is, to characteristics which might be described as 'psychological'. It is not easy to ascertain in the case of individual users the degree to which the turtle, the computer, the language itself, even the procedures, all of which in various degrees partake of personification within the literature of Logo, are actually anthropomorphized as part of the Logo experience. However, particularly in view of the confusion often experienced by younger children regarding the boundaries between the animate and the inanimate [7], it seems overoptimistic to expect that a simple caution such as quoted below would suffice to set the record straight.

When we talk about Logo as if it were a person who 'knows', 'understands', 'wants to help you', etc., we do so because it helps us think about solving problems with the computer. We know that Logo isn't really a person, but if we visualise Logo as a personality, it helps us understand its behaviour at a number of tricky points $[8$, p. 8].

\section{The 'social' computer}

Where a significant degree of personification is accepted, albeit tacitly, in learning situations, it is reasonable to question the status of the 'social' computer as an intellectual collaborator. In the case of Logo, for instance, what role might the computer, as manifested through the Turtle, be presumed to play in the social dynamics which mediates classroom learning? On the positive side it may be perceived as nonjudgemental, as lacking in bias and as demonstrating with consistency a particular form of rationality. In addition it can be seen to provide, both through its actions in response to commands and through the language of error messages, immediate feedback and a degree of practical support. On the other hand the Turtle as a participant in arguably social interactions is deficient in a number of qualities which might be regarded as particularly important in situations in which learning is being negotiated. It clearly lacks the rich complexity of prior understandings and relationships possessed by even the youngest of human participants, it lacks flexibility in language use, it is devoid of anything approaching human selfawareness or insight. In relation specifically to learning it lacks the capacity to modify its own cognitive structures and processes. Furthermore it can be argued strongly 
that the model by which the turtle 'learns' is fundamentally nonconstructivist in nature, making it a highly inappropriate role model in the classroom situation. When these issues are considered in conjunction with other more general aspects of the way in which the technology is commonly perceived, particularly in relation to the authority which is often assumed to be implicit in any computer generated output, it can be seen that the personified computer might well be viewed as a somewhat problematic participant in classroom interactions.

\section{The 'personal' interface}

A growing awareness of these issues in the wider world of computing has a great deal to contribute to our consideration of their implications in the classroom. Recent developments in interface design as exemplified by the ideas of Laurel [9], or by the well-known character of Phil, which has featured in a number of Apple Computer's futuristic promotional videos, indicate the degree to which the personification of computers through their interfaces is often felt to be advantageous to the user in a number of respects. Many writers, including Laurel, see personification as both natural and desirable in that it enables us to make use of our considerable experience of interacting with other human beings in understanding, predicting, and controlling computers for our own ends. On the other hand both she and others also acknowledge the complexities and possible problems which could arise from too complete an acceptance of personification. A number of these concerns relate to the role of computers within relationships, particularly in regard to the extent to which they are regarded as possessing superior 'expertise', and to the 'tone' of the language through which they interact with the user. Andersen [10], for instance, warns that some users are threatened by the potential for interpersonal intimacy implied by a heavily personified interface. This relates to concerns expressed by Marcus regarding the need for computers to behave verbally with what he terms 'decorum', that is, 'saying the right thing in the right way at the right time to the right person' [11, p. 5]. Such factors are clearly of great importance in defining the status of the computer within the social milieu of the classroom. For instance, as mentioned above in connection with Logo, in situations which might otherwise be described as collaborative, the 'authority' of the computer can be seen as intruding, perhaps to an even greater extent than traditional authority figures such as teachers. The other side of this question relates to the degree to which the control and domination exercized over a significantly personified entity, both in the classroom and in relation to computing in general, might be perceived as encouraging inappropriate relationships with one's fellow human beings. As Laurel writes in relation to 
her conception of 'agents', aspects of the computer interface which function in a more or less personified way in response to the demands of the user [9]:

Perhaps a more thought-provoking objection to agents rests on an ethical argument which goes something like this: if an agent looks and acts a lot like a real person, and if I can get away with treating it badly and bossing it around without paying a price for my bad behaviour, then I will be encouraged to treat other, 'real' agents (like secretaries and realtors, for instance), just as badly (p. 356).

\section{The 'dying' computer}

Other writers such as Gelernter [12, p. 210] raise similar questions, for instance in relation to the eventual disposition of significantly personified manifestations of computers when their term of usefulness is at an end. While such concerns might appear extreme to the point of frivolity at this point, they are clearly a logical extension of some of the 'ethical' issues which are hinted at by current developments in computing technology. One might well imagine, for instance, that in a hypothetical virtual reality or cyberspace environment where such agents might be merged in significant sense with the personality and intentions of the user, and where interactions with other such entities would have consequences in the wider world, such considerations would be very real. The seriousness with which these issues are debated can be contrasted with the airy insouciance with which Solomon was able to write, as recently as 1986, in relation to the personification of aspects of the Logo environment, "We can conjure up these little people whenever we need help. We can simulate them, that is, be them for a while, talk to them dispose of them!" [6, p. 120].

It is apparent from a number of sources that an increasing number of educators and theorists are interested in exploring seriously the role of the computer as a participant in relationships with its users which can be characterized in a significant sense as personal or social. Further, as Marcus suggests [11]:

The power and influence people invest in (or derive from) computers seem to make these issues especially pertinent. The impact of the computer on a person's selfimage, sense of power, and affiliations with others is a major aspect of the computer revolution, and these dimensions of the affective domain deserve and require special attention in attempts to develop the personable computer. (p. 5). 


\section{CONCLUSION}

While it might be objected that personification is by no means universally favoured as the ideal form of human-computer interface, indeed there are many proponents of alternative images such as that of computing 'space', it can be argued strongly that a degree of personification is the inevitable consequence of certain aspects of the way in which computing technology has developed, particularly in relation to the concept of 'artificial intelligence' and to the extent to which language is involved at many levels in the interface. This being the case, the role of the computer in relation to the social and cognitive interactions which take place in the classroom, is of considerable interest.

\section{REFERENCES}

1. Haraway, D. (1985) A manifesto for cyborgs: science, technology and socialist feminism in the 1980's. Socialist Review 80, pp. 65-107.

2. MacCormac, E.R. (1986) Men and machines: the computational metaphor, in Mitcham, C. and Huning, A. (eds) (1986), Philsophy and Technology 11: information technology and computers in theory and practice, Kluwer Academic Publishers, Dordrecht, The Netherlands.

3. Winner, L. (1986) The Whale and the Reactor: A Search for Limits, in an Age of High Technology, University of Chicago Press, Chicago.

4. Shirk, H. N. (1988) Technical writers as computer scientists: the challenges of online documentation, in Barrett, E. (ed.) (1988), Text, ConText and Hypertext: writing with and for the computer, MIT Press, Cambridge, Massachusetts.

5. Dennett, D.C. (1981) Brainstorms: Philosophical Essays on Mind and Psychology, MIT Press, Cambridge, Massachusetts.

6. Solomon, C. (1986) Computer Environments for Children: A Reflection on Theories of Learning and Education. MIT Press, Cambridge, Massachusetts.

7. Turkle, S. (1984) The Second Self: Computers and the Human Spirit. Granada, London.

8. Watt, D. (1984) Learning with Apple Logo. McGraw Hill, New York. 
9. Laurel, B. (ed) (1990) The Art of Human Computer Interface Design. Addison Wesley, Reading, Massachusetts.

10. Andersen, P.B. (1990) A Theory of Computer Semiotics. Cambridge University Press, Cambridge.

11. Marcus, S. (1992) Decorum by design: From HAL to pal. The Designers' Forum 1 (1).

12 Gelernter, D. (1991) Mirror Worlds. Oxford University Press, New York. 